\title{
An Introduction to a Novel Intervention, "This is My Story", to Support Interdisciplinary Medical Teams Delivering Care to Non-Communicative Patients
}

\author{
Elizabeth Tracey ${ }^{1} \cdot$ Thomas Crowe $^{1} \cdot$ Jason Wilson $^{1} \cdot$ John Ponnala $^{1}$. \\ Joshua Rodriguez-Hobbs ${ }^{1} \cdot$ Paula Teague $^{1}$
}

Accepted: 31 July 2021 / Published online: 12 August 2021

(c) The Author(s), under exclusive licence to Springer Science+Business Media, LLC, part of Springer Nature 2021

\begin{abstract}
This set of three case studies portrays a unique intervention undertaken at The Johns Hopkins Hospital in response to the COVID-19 pandemic with a goal to reduce the impact of absentee visitors during patient care on physicians, nurses, and the patient's loved ones. The intervention, known by the acronym TIMS, "This is My Story", involves a chaplain-initiated telephone call to a loved one, someone who has been identified by the patient as part of their care discussions, of hospitalized patients who have difficulty with communicating to the medical team. The call is recorded then edited for conciseness, and attached to the electronic health record for the entire medical care team to hear. The focus of the chaplain lead conversation with a loved one centers around gathering and presenting information about the patient as a person. Medical team members listen to the edited audio file either on rounds or by utilizing a hyperlink in the electronic health record (EHR). The audio file is two minutes or less in length, as this is the optimal size for comprehension without overburdening the care provider. While conducting the interview, there is an opportunity for chaplains to provide spiritual and emotional support to loved ones and medical staff, contributing substantively to patient care, as is illustrated in the case studies.
\end{abstract}

Keywords Chaplain interview - COVID-19 interventions · Visitor restrictions · Response to isolation · This is my story · Loved one's absence $\cdot$ Moral injury · Moral distress $\cdot$ Pandemic $\cdot$ Telechaplaincy

Elizabeth Tracey

Etracey@jhmi.edu

1 Department of Spiritual Care and Chaplaincy, Johns Hopkins Hospital, 600 N Wolf Street, Baltimore, MD, USA 


\section{Introduction}

The COVID-19 pandemic has resulted in considerable moral distress (Morley, 2020), and moral injury for medical staff (Williamson, 2020a; b), who are called upon to risk their welfare and possibly that of their loved ones in caring for patients with COVID-19. They must deliver care for which no algorithm exists and witness unprecedented rates of death, often taking place with no family or loved ones present. As Menon (2021) describes COVID-19 challenged health care workers with a "sense of guilt, anguish, helplessness, uncertainty, and powerlessness when one is fighting something on such a powerful scale with limited resources and no definite end in sight" (p. 1). This intervention intends to give providers pertinent information about the patient as a person and assist in making difficult bioethical choices while returning the ability to make an informed decision to the care provider as they now have a more wholistic portrait of the patient.

A conversation between a provider and a chaplain on the medical intensive care unit (MICU) at The Johns Hopkins Hospital demonstrated just such distress, as described in the presentation of case 1 with Beverly Johnson. Very early in the pandemic, the medical provider revealed that he was found taking care of COVID-19 patients challenging because "they're all intubated, sedated, often prone, and there's no family at the bedside telling me their story." This provider's story was echoed by others and indicated a significant gap in information which contributed to the provider's troubling sense of loss of humanity in medicine. Awareness of the moral distress and injury and these conversations led to the development of the TIMS project as a measured response.

The board-certified chaplain working on the MICU who heard these stories has a background in broadcast journalism. She suggested that she could reach out to the family and learn something of the patient's story and could also record the interview. Being familiar with the workflow on the MICU, she was also sensitive to time constraints as well as when and where an audio file might be utilized. The provider in collaboration with the chaplain reviewed and agreed upon the intervention, and the TIMS project was initiated in April 2020. Patients were referred by an administrator on the unit, in consultation with nurse leaders and medical care providers. The phone calls were made to those individuals who were highlighted as the patients' emergency contacts.

\section{Methods}

\section{Site}

The locale for this study was the medical intensive care unit (MICU) at the Johns Hopkins Hospital, and the implementation of this intervention was in consultation with two of the attendings for this unit. The chaplain that initiated this method of interviewing the family is familiar with the staff and structure of this unit as she 
is often embedded in interdisciplinary teams regarding patients at the end of life with no loved ones able to be present and she, or a representative of the program, can be present as the patient transitions. This qualitative intervention was started during the early phases of biocontainment for this unit as the COVID-19 pandemic began, in late March 2020, and addressed perceived stress and anxiety by the medical team as they were not able to gather contextual information about the patient as they had previously done before strict visitor restrictions.

\section{Participant Population}

Although this paper only discusses three of the TIMS audio files, at the time of writing this article, there have been over four hundred referrals for this intervention across the entire hospital. In preliminary data analysis, there have been no trends for gender, age, ethnicity observed but this is not a self-referral study, but it is referred by staff on the unit as the patients are admitted to the ICU without visitors able to be present. Later analysis may demonstrate a link to certain variables, however, and in conjunction with a discussion of the providers on their usage of the audio file may elicit additional insights.

The presented case studies are a convenience sample, as outlined in Table 1 below and are not a representative sample of the hospital census. The author presents the following case studies as exemplars for the collection and use of the TIMS audio file. The overall discussion for method and results are identified in the three case studies but none are meant to exemplify all the results or methods.

Participants who are selected to receive the TIMS audio file are required to meet specific inclusion and exclusion criteria. This has been slightly revised through the pilot phase, the first 6 months, as the referrals increased and the labor to conduct the interview needed to be appropriately distributed. The first criterion for the patient is they have a communication difficulty (e.g., intubation, incapacity, etc.) and they are not able to share contextual information with the medical team. The second criterion for the intervention is the patient does not have any, or infrequent visitors, present for the medical team to talk with while they are providing care. This criterion is shifting now as the hospital is reducing visitor restrictions, however, it is still our premise that patients who are not able to communicate for themselves cannot solely rely upon loved ones for the 24-h tempo of an ICU to relay their story. Lastly, for inclusion, their length of stay is greater than three days as the recording and editing phase typically takes $72 \mathrm{~h}$ to be completed.

Table 1 Case study demographics $(n=3)$

\begin{tabular}{|c|c|c|c|}
\hline $\begin{array}{l}\text { Self-identified } \\
\text { gender }\end{array}$ & Age & Race & Principle condition \\
\hline Female & 74 & Caucasian & COVID-19 \\
\hline Male & 33 & Caucasian & Congenital cerebral palsy \\
\hline Male & 53 & Caucasian & $\begin{array}{l}\text { Liver transplantation } \\
\text { Post-Idiopathic Steato- } \\
\text { hepatitis }\end{array}$ \\
\hline
\end{tabular}




\section{Logistics for Audio File}

When determining a prospective patient for the TIMS audio file, it was done in collaboration with the customer care service representative (CCSR) and/or the attending physician or charge nurse. Once the referral is generated, the chaplain reaches out by telephone to a loved one, as identified in the electronic health record (EHR), to initiate the interview or to schedule a time that is most convenient to the loved one. If the loved one is identified as a non-English speaker, an audio translator is contacted and brought into a conference call before calling the family member as they will start the conversation in the preferred language of the loved one. A voice recording application on a cell phone or other mobile device is used to record the conversation, while the phone call is made using another telephone or computer. The recording is then given to medical student editors who utilize an audio editing software program to edit the file to 2 min or less and return it for verification by the interviewing chaplain to ensure they have captured the crucial discussion points during the discussion.

During the discussion with the loved one, a structured theme is used for consistency and to verify that similar information is collected during the discussion. There is an open-ended interview section toward the ending of the interview where they can express their wish for the patient or what they feel the medical team should know. Routinely, the discussion starts with the loved one being asked a generic question of "who is this person" with some examples such as telling us if they have pets, sports teams, and favorite foods. This allows the interviewee to get more familiar and comfortable with the interview process, and then, it progresses into asking them to "paint a picture" of the person for someone who doesn't know them such as how they spend their free time or their current relationships in their life. As the interview is reaching the mid-point, the loved one is asked if they were present with the patient, "what would you want to say to them if you were face to face?"

Often, this is the part of the interview that goes beyond a subjective detail of their life and begins to focus on more details of the person whom we are speaking about. This part of the interview is used for end of life when patients can't have visitors, as this was the case in early pandemic visitor restrictions, or as they are being oriented from sedation. Lastly, in the more open-ended portion of the discussion, it is asked about their hopes or fears regarding their loved one and if there is anything that the loved one thinks the medical team caring for them would need to know.

The chaplain reviews the edited version of the interview then posts it to a secure media server website and obtains a hyperlink to place in the medical chart. The hyperlink is placed so that all members of the medical care team are advised of the availability of the TIMS recording and can access the file. Unit rounds and nursing huddles are attended by the chaplains to discuss the project and answer any questions that might arise.

Medical students get the opportunity to hear a patient's story told from the perspective of the family and are given the directive to edit with the adjective "tender" in mind. They are reminded that they are creating the narrative of someone's beloved. Thus far, those performing the editing are being exposed to a more narrative format for collecting a patient's details and expressed appreciation for this as 
part of their education. Medical students and others are specifically trained to edit the files and return the audio files within a 24-h timeframe.

\section{Staff}

Throughout the implementation phase of this study, it was discussed whether the chaplain is the appropriate person to conduct this interview. The author's premise is that this is appropriate given the "the link between spirituality and moral injury has in some cases been ignored or undervalued while a number of other researchers have identified its relevance" (Hodgson \& Carey, 2017, p. 1220). When "responding to moral injury should be through 'specific communal practices' rather than a 'medical model', to allow for an honest and real narration and recovery from moral injury experiences" (Hodgson \& Carey, 2017, p. 1221). The TIMS process, interview, and discussion with the medical staff give space to discuss with the provider their anxieties or fears and begin to put a remedy in place. The chaplain is the person on the interdisciplinary team who is best suited to be able to have this emotional and spiritual discussion with the medical staff as they are uniquely trained and outside of the chain of command for that person, thus allowing them to accurately portray their true feelings.

\section{Results}

Thus far the observed results have been three-fold, impacting the medical staff hearing them and the patients or loved ones in which the files are created about. A formal mixed method, quantitative and quantitative study, has recently been implemented to look at the impact and efficacy for the medical team; however, preliminary anecdotal evidence is available. Often the feedback is an appreciation for "humanizing" the patients during a time wherein medical care the personhood is being lost due to pandemic fatigue and a perceived loss of connection with the patient. One nurse commented that it "gives them something to talk about with their intubated patients... and to see a smile on their face." (MICU nurse, personal communications, May 4, 2021) Patient reactions are often described as different with the medical team as they are "transformed" after listening to the TIMS files. They describe previous communications as businesslike and clinical, while those subsequent were "personal" and "human."

During the planning stages of this intervention and discussion with the medical staff, one attending physician initially shared that he was "not convinced" that TIMS files would have much impact but agreed to use them on medical rounds and would evaluate. After a few days, he began requesting TIMS files for all his patients after witnessing their benefit for the medical team and as seen in the case study below, requested the file played for Beverly at the end of life. 
Case Study 1: Beverly Johnson

Beverly Johnson (release obtained) was a 74-year-old female admitted to The Johns Hopkins Hospital after acquiring COVID-19 infection at a long-term care facility, where she had been receiving rehabilitation following a procedure. She was intubated and sedated with severe COVID-19 disease. Her attending physician asked the chaplain to obtain a TIMS file for her. Beverly's contact was identified as her sister, Barbara Johnson (BJ)

Chaplain: Hi, is this Barbara? My name is Elizabeth Tracey, and I'm part of the team at Johns Hopkins helping to take care of your sister. I'm calling to ask you to take part in a project we are doing in the MICU to help the medical team know a bit more about Beverly as a person. As you know under normal circumstances you would be here at the hospital telling us about Beverly, but because we are not allowing visitors you aren't here, so I'm hoping you would be willing to spend about $10 \mathrm{~min}$ with me telling me a bit about her. Would that be okay? With your permission, I will record our conversation

BJ: (a very articulate and passionate woman): Yes, indeed. I'm not sure where to start...

Chaplain: Well, I usually start with dogs. Does Beverly have a dog?

BJ: No, no dog. But cats. Two cats, and we're going over to her place to take care of them for her...

In addition to the audio file for the medical team, Barbara also recorded one for Beverly, which nursing played for Beverly several times and at the end of life

Both the attending and the chaplain were present when Beverly was taken off the ventilator and died peacefully within a few minutes. The portion of the interview where the loved one is asked about "what would you say if you were face to face..." provided closure in this instance for Beverly's sister.

Chad was a patient in the fall of 2020 when the hyperlink for the TIMS audio interview file began to be included in the EHR. The chaplain sought to understand how the medical staff was accessing the file, so while on routine visits to the MICU would ask members of the team if they had heard the file, and if not, to listen to it with them. One of these was a nurse named Alexandra (A), and this was an exploration to assess the impact and use of the TIMS audio file for the medical staff.

Case Study 2: Chad

Chad (release obtained) is a 33-year-old male with cerebral palsy. He was admitted to The Johns Hopkins Hospital MICU with an inability to maintain his body temperature and some respiratory difficulties. The chaplain reached out to Chad's mother (release obtained) at the request of the medical team

Chaplain: Hi, I'm Elizabeth, one of the chaplains. I'm just wondering if you've had a chance to listen to the TIMS file on this patient

A: No, I don't even know what that is

Chaplain: Here, it's right here in the Sticky Note (signing onto the computer and the EHR). You just copy and paste the link into a browser (doing so). It's less than 2 min long. Do you have time to listen?

A: Sure. (We listened to the file)

A: Wow, that was really wonderful. Thank you for sharing that with me. There's a lot there I didn't know about Chad

Chaplain: You're welcome! What resonated for you in the file?

A: I think that his Mom says he's her joy. That's hard to believe. It's so tough caring for someone so disabled. I would have thought she would be happy he's here so she gets a break! It really helps me understand the family a lot better, and makes the whole story much more human

Chaplain: I'm so glad you feel that way 


\section{Case Study 2: Chad}

A: Yes, I hope you do them for all my patients. You know, besides here I also work in pediatric hospice, and that story reminds me of what I hear parents say about their kids all the time- that in spite of their illness and the challenges of caring for them, they are so precious to them

Chaplain: Yikes, pediatric hospice! That's a tough call

A: (nodding and smiling) Yes, most people say that. But I would say that lots of what I do with them, like watch movies or play Wii, wouldn't be called work anywhere else

Chaplain: Thank you for sharing that. You've given me a new perspective too!

This experience with Alexandra demonstrates how TIMS files can also enable chaplains on the unit to be present for staff, a critical role right now as burnout is rife and isolation almost universal.

Lastly, the medical team on the MICU requested a TIMS file which was recorded with Jeff's brother, Ron. The file was utilized by multiple medical teams as Jeff was transferred from the surgical intensive care unit (SICU) to the transplant unit and finally to rehabilitation. This example will be how the patients are impacted through the creation of the TIMS audio file.

Case Study 3: Jeff

Jeff (release obtained) is a 53-year-old male admitted to the MICU at The Johns Hopkins Hospital with acute liver failure secondary to idiopathic non-alcoholic steatohepatitis (NASH). Jeff was critically ill, requiring intubation and bedside dialysis as his kidneys as well as his liver were failing. The medical team evaluated him for transplant but was concerned that he might not survive. Jeff began to improve enough to survive surgery and because he had a rare blood type, a liver became available. He underwent surgery and received a liver, and the chaplain continued to follow him for three months until he was discharged

During one of our visits, the chaplain told Jeff $(\mathrm{J})$ about the TIMS project and asked him if he would like to listen. He said yes, so we listened together

$\mathrm{J}$ : Well that's an interesting project

Chaplain: What do you find interesting about it?

J: (laughing) I guess it helped the medical staff understand why I only want bagels!

Chaplain: (laughing). Yes, you do have some interesting food preferences, and I'm glad they understand them now

J: My brother was right about my nephews. I miss them so much! and my dog. They keep trying to put my dog on FaceTime but he just doesn't get it. He doesn't even look at the phone when I talk. After all this time I don't even think he remembers who I am

Chaplain: I bet he remembers

J: Yeah. I guess I never knew how much my brother cares. He keeps telling me they aren't allowed to come here, and I know that's true. It's just so hard to not see anyone. Except you all, I mean. And I am so grateful to everyone. The nurses have been so fantastic, and I really enjoy your visits. But I would really love to see my family. It's good to know Ron feels the same way

While he was hospitalized, the chaplain routinely played the TIMS file for his nurses. One nurse in the SICU listened and then became tearful. She confided that she, too, had a similar medical condition. She was attempting to do everything she could to avoid liver transplantation, and she told me the file allowed her to view Jeff with greater compassion. 
Perhaps the most valuable listening came for Jeff himself. Because his family lived about three hours away from the hospital and the no visitor mandate remained, Jeff's family had not come to see him during his lengthy hospitalization. As he was recuperating Jeff also experienced confusion and felt abandoned by his family, who were very important to him. Upon hearing this audio file, realizing that his family was experiencing a similar longing to be present with him, possibly unable to explain this, it provided him comfort. So far there is only anecdotal evidence of the impact or efficacy of the TIMS audio intervention; however, once the quantitative and qualitative study concludes, there can be a much deeper dive into the use of this file and its effect during the COVID-19 pandemic.

\section{Next Steps}

Spiritual Care and Chaplaincy extended the TIMS initiative to other units and hospitals in the Hopkins system in September 2020. The Spanish language, as well as other language recording and translations, were initiated. The chaplain team provides administrative support and helps to incorporate members of teams desiring to employ TIMS in their respective departments throughout the health system. TIMS files are now being recorded by board-certified and/or CPE trained chaplains at both The Johns Hopkins Hospital and Johns Hopkins Bayview Medical Center for patients with communications limitations, especially those on ventilators.

We are currently in the data-gathering phase of a study evaluating the impact of TIMS on all persons comprising the medical team caring for a patient. After the study, we hope to have identified best practices for integrating audio files into patient care with the primary goal of easing clinician burden and bringing greater humanity to medical practice.

\section{Limitations}

This study is still in the initial stages of being conducted and the assessment and evaluation phase has just begun internally. The demographic representation and institutional implementation may be different as this is being conducted in a medical intensive care unit (MICU) within an academic hospital. We do not know if other units within this hospital will desire this file or use it as has been seen on the MICU. Another factor is the reduction of COVID-19 isolation units for patients, and we are unsure if this intervention will continue as it is currently being used has once the visitor restrictions are lifted.

As discussed in the population section, a convenient sample has been presented but doesn't reflect the demographic composition of the hospital in which this study is being conducted. Later inclusion of a representative population is being implemented as this pilot portion is being completed and will include a much broader subject population. 


\section{Conclusion}

The collective vision for TIMS has expanded and we believe such recordings merit a place in a human-centered approach to the care of patients and families. As the efficacy of this intervention is assessed, there are plans to expand it to all patients with communications limitations, which may mean at some point almost all inpatients as well as those seen via telemedicine.

The future directions and evaluations of this approach will be based upon how the clinicians use the file and if they find it useful in such instances as; reducing their burden to communicate with families that are separated due to visitor restrictions and allow them to have a personal connection with patients that are not able to speak for themselves or if there are language barriers that prevent a patient's story from being fully communicated.

Funding Funding was received by Johns Hopkins COVID Relief Fund Grant number $(\$ 50,000)$.

\section{Declarations}

Conflict of interest We have complied with all ethical standards in conducting this research and we have no conflicts of interest to report.

\section{References}

Hodgson, T., \& Carey, L. (2017). Moral injury and definitional clarity: Betrayal, spirituality and the role of chaplains. Journal of Religion and Health, 56, 1212-1228. https://doi.org/10.1007/ s10943-017-0407-z

Menon, B., Narayan, S. K., \& Bhade, S. (2021). COVID-19, moral injury and the Bhagvad Gita. Journal of Religion and Health, 60, 654-662. https://doi.org/10.1007/s10943-021-01210-Z

Morley, G., Sese, D., Rajendram, P., \& Horsburgh, C. (2020). Addressing caregiver moral distress during the COVID-19 pandemic. Cleveland Clinic Journal of Medicine. https://doi.org/10.3949/ccjm.87a. $\operatorname{ccc} 047$

Tracey, E. (Executive Producer). (May 5, 2020) This is My Story (Audio recording) The Johns Hopkins Hospital. https://s3.amazonaws.com/JHM-Podcasts/wp-content/uploads/2021/02/07124242/bever ly0505mp3.mp3

Tracey, E. (Executive Producer). (December 28, 2020) This is My Story (Audio recording) The Johns Hopkins Hospital. https://s3.amazonaws.com/JHM-Podcasts/wp-content/uploads/2021/02/07124 127/chad1029.mp3

Tracey, E. (Executive Producer). (October 18, 2020) This is My Story (Audio recording) The Johns Hopkins Hospital. https://s3.amazonaws.com/JHM-Podcasts/wp-content/uploads/2021/02/07154259/ russell1029.mp3

Williams, R., Brundage, J., \& Williams, E. (2020a). Moral injury in times of COVID-19. Journal of Health Service Psychology, 46, 65-69. https://doi.org/10.1007/s42843-020-00011-4

Williams, V., Murphy, D., \& Greenberg, N. (2020b). COVID-19 and experiences of moral injury in frontline key workers. Occupational Medicine, 70(5), 317-319. https://doi.org/10.1093/occmed/kqaa052

Publisher's Note Springer Nature remains neutral with regard to jurisdictional claims in published maps and institutional affiliations. 